\title{
ALK and EGFR expression by immunohistochemistry are associated with Merkel cell polyomavirus status in Merkel cell carcinoma
}

\section{Veija, Tuukka}

2019-05

Veija , T , Kero , M , Koljonen , V \& Böhling , T 2019 , ' ALK and EGFR expression by immunohistochemistry are associated with Merkel cell polyomavirus status in Merkel cell carcinoma ' , Histopathology , vol. 74 , no. 6 , pp. 829-835 . https://doi.org/10.1111/his.13815

http://hdl.handle.net/10138/308748

https://doi.org/10.1111/his.13815

unspecified

acceptedVersion

Downloaded from Helda, University of Helsinki institutional repository.

This is an electronic reprint of the original article.

This reprint may differ from the original in pagination and typographic detail.

Please cite the original version. 
Article type : Original Article

20.12.2018

\section{ALK and EGFR expression by immunohistochemistry are associated with Merkel cell polyomavirus status in Merkel cell carcinoma}

Tuukka Veija ${ }^{1}$, Mia Kero², Virve Koljonen ${ }^{3}$, Tom Böhling ${ }^{1}$

${ }^{1}$ Department of Pathology, University of Helsinki, Helsinki, Finland

${ }^{2}$ Department of Pathology, University of Helsinki and HUSLAB, Helsinki University Hospital, Helsinki, Finland

${ }^{3}$ Department of Plastic Surgery, University of Helsinki and Helsinki University Hospital, Helsinki, Finland

Running title: ALK and EGFR expression associate with MCV status in MCC

Conflict of interest statement: Each author declares no financial or personal conflicts of interest with regards to the data presented in this manuscript.

This article has been accepted for publication and undergone full peer review but has not been through the copyediting, typesetting, pagination and proofreading process, which may lead to differences between this version and the Version of Record. Please cite this article as doi: 10.1111/his.13815

This article is protected by copyright. All rights reserved. 


\section{Correspondence to:}

Tuukka Veija, Department of Pathology, P.O. Box 21, FI-00014 University of Helsinki, Finland Email: tuukka.veija@helsinki.fi

\section{Abstract}

Aims:

Merkel cell carcinoma, a rare cutaneous neuroendocrine tumor of the skin, can be categorized into two groups according to Merkel cell polyomavirus (MCV) presence. MCVnegative tumors are more aggressive and frequently associated with gene mutations. Some of the genes are potential therapeutic targets. We have previously reported EGFR mutations in 6/27 MCC tumors and overexpression of ALK and EZH2 at mRNA level in MCC tumors. In this study, we sought to determine expression of ALK, EGFR and EZH2 in MCC samples and assess their correlation to MCV status and clinical parameters.

Methods and results:

Tissue microarrays were utilized and stained with primary antibodies. Staining data was statistically compared to patient sex, tumor location and development of metastasis and MCC specific death. 112 tumors and their corresponding patient data were included. We found strong expression of ALK in 51\% and strong expression of EZH2 in $76 \%$ of the tumors. There was evident correlation of ALK expression with MCV-positivity. Expression of EGFR was infrequent presenting only in $7 \mathrm{MCV}$-negative tumors. None of the proteins associated with development of metastasis or MCC specific death.

Conclusions:

ALK and EZH2 expression are frequent in MCC and ALK expression correlates to MCV positivity. EGFR positive tumors might respond to EGFR inhibiting treatment.

Key words: Merkel cell carcinoma, Merkel cell polyomavirus, Immunohistochemistry

This article is protected by copyright. All rights reserved. 


\section{Introduction}

Merkel cell carcinoma (MCC) is an aggressive neuroendocrine skin cancer categorized into two groups according to the presence of the Merkel cell Polyomavirus (MCV). ${ }^{1-4} \mathrm{MCC}$ has a tendency to metastasize; it spreads to the lymph nodes more often than other skin malignancies. ${ }^{5}$ Notably, MCV-negative tumors seem to be the more aggressive as compared with MCV-positive tumors. ${ }^{6,7}$

We have previously studied MCC tumors for aberrations in cancer-related genes utilizing targeted next-generation sequencing (NGS). The most intriguing findings of our NGS studies were mutations of the EGFR gene in $22 \%$ of $27 \mathrm{MCC}$ tumors, and overexpression of $A L K$ and EZH2 at mRNA level in MCC tumors as compared to normal skin. ${ }^{8,9}$ Most of the EGFR mutations we detected are previously reported in non-small cell lung cancer ${ }^{10}$ and at least one of the mutations has been reported to be responsive to EGFR inhibitor treatment in lung adenocarcinoma. ${ }^{11}$ EGFR and ALK are transmembrane receptor tyrosine kinases involved in many types of cancer, for example non-small cell lung cancer. ${ }^{12-16}$ ALK is named after its involvement in a fusion protein discovered in anaplastic large cell lymphomas. ${ }^{17}$ There is no evidence of activating ALK mutations ${ }^{8,18,19}$ or fusions ${ }^{9,20}$ in previous literature regarding $\mathrm{MCC}$ and therefore the mechanism of ALK overexpression is unknown. EZH2 is an enzyme that silences gene function by promoting DNA heterochromatin formation. It is normally expressed during fetal development, but expression of EZH2 is found in various types of cancer, and the role of EZH2 inhibitors in cancer therapy is under investigation, with multiple ongoing preclinical projects ${ }^{21}$ while at least one phase I study regarding B-cell lymphoma and epithelioid sarcoma is completed. ${ }^{22}$

This article is protected by copyright. All rights reserved. 
MCV-positive and MCV-negative tumors have distinct pathological characteristics; therefore it is rational to seek for molecular aberrations in subsets of MCC tumors that would potentially benefit from certain targeted therapy. Mutational studies indicate that especially MCV-negative tumors are associated with more gene mutations, the majority of which are caused by UV radiation. Some of these mutated genes are considered potential targets of therapy for certain MCC tumors. ${ }^{8,18,19,23}$ Based on our previous results, in this current study we sought to determine expression of ALK, EGFR and EZH2 by immunohistochemistry in a large tumor cohort and assess their correlation to the tumor MCV status and potential prognostic or therapeutic value of these proteins.

\section{Materials and Methods}

The Ethics Committee of Helsinki University Hospital approved the study. The Ministry of Health and Social Affairs granted permission to gather patient data, and the National Authority for Medicolegal Affairs to collect and analyze tumor samples. Clinical data gathered for this study included patient age at diagnosis, sex, location of primary tumor and information on whether the patient developed metastasis or died for MCC. The clinical details were gathered for these patients as they were in $12^{\text {th }}$ June 2013 and no further follow-up was conducted.

Tissue micro arrays (TMA) were constructed from our nationwide pool of formalin-fixed paraffin-embedded (FFPE) MCC tumor samples. MCC diagnoses were confirmed by clinical characteristics and microscopic morphology compatible with cutaneous MCC and by 
immunohistochemistry positive for CK-20 and negative for TTF-1. MCV status of the tumors was determined by quantitative PCR as described in detail elsewhere. ${ }^{24}$ From the FFPE tumor samples, representative tumor regions were first defined from H\&E-stained sections and marked. A 0.6- $\mathrm{mm}$ tissue cores of each tumor sample was inserted into an empty well on the tissue array block. Two cores per tumor was included at initial construction. $3 \mu \mathrm{m}$ sections were cut from the TMA block and processed for immunohistochemistry.

For immunohistochemical stainings, a Ventana Benchmark Ultra instrument (Roche, Tucson, AZ, USA) was utilized for ALK-1 (clone D5F3, Roche 790-4794) and EGFR (clone 5B7, Roche 790-4347) RTU antibodies. For pretreatment of these antibodies we used Cell Conditioning 1 buffer, pH8.5, (Roche 950-124), $64 \mathrm{~min}$ in $98^{\circ} \mathrm{C}$. The incubation time for ALK-1 was 28 $\min / 36^{\circ} \mathrm{C}$ and for EGFR $20 \mathrm{~min} / 36^{\circ} \mathrm{C}$. The multimer based detection kit, OptiView, (Roche 760-700), was used to detect the antibodies. An amplification step was added for both protocols by using separated an amplification kit (Roche 760-099). Lung adenocarcinoma tissue with EML4-ALK translocation confirmed by FISH as well as anaplastic large cell lymphoma tissue with NPM1-ALK translocation confirmed by FISH was used as a positive control for ALK staining while the negative controls were lung adenocarcinoma and $A L C L$ samples without the mentioned chromosome rearrangements. For EGFR, normal epidermis as well as human placenta were used as positive controls. The EZH2 staining (clone 11/EZH2, BD Transduction 612666, USA) was performed in the LabVision immunostainer (Labvision, CA, USA). Antigen retrieval was done by using the Tris-EDTA buffer, pH 9.0 (in a PT- module for 20 minutes in $98^{\circ} \mathrm{C}$ ). For detection, we applied the polymer-based detection system (Envision, K5007, Agilent, USA). DAB was applied as a chromogen for all of the antibodies and the slides were stained with hematoxylin (Mayer, S3099, Agilent, USA).

This article is protected by copyright. All rights reserved. 
Immunohistochemical stainings were interpreted by two researchers (TV and TB). Individual samples on the TMA slides were disqualified if the sample was partly or completely missing or technically excluded during the construction process, if the sample did not include tumor tissue or if the MCV-status of the tumor was unknown. The exact number of included samples varied from 110-111 between stained TMA slides, but altogether there were 112 tumors that had successful staining with at least 2 of the antibodies and therefore were included in the further analysis. Regarding ALK and EZH2 IHC, there was large variation of staining intensity between individual samples, and thus based on the intensity of staining, expression of ALK and EZH2 was interpreted as either negative, weak positive or strong positive. (Figures 1 and 3) In contrast, EGFR expression was simply interpreted either negative or positive. (Figure 2)

Statistical analysis to correlate protein expression to MCV status and clinical data was done with the Chi-Squared and Fisher's exact test. The Chi-Squared test was also utilized to correlate the presence of metastasis during the follow-up to MCC specific death. To analyze the relationship of protein expression and MCC-specific death, the survival was estimated with the Kaplan-Meier method and the logrank test was used to compare the survival between the groups. MCC specific survival was calculated from the date of diagnosis to date of death from MCC. P-values less than 0.05 were considered significant. Statistics were done with NCSS statistical software (NCSS, LCC.). The Kaplan-Meier plots are presented in supplementary material.

This article is protected by copyright. All rights reserved. 


\section{Results}

This study included TMAs containing 112 Merkel cell carcinoma tumor samples with respective clinical data. Of those 112 patients, 30 (27\%) were males and 82 (73\%) females. The mean age of the patients was 78 years. Over half of the tumors, 60 (54\%) were located in the head and neck region. 31 tumors were MCV-negative (28\%) and 81 MCV-positive (72\%). Distant metastasis was present in 28 cases (25\%), while 11 cases $(9.8 \%)$ had only local lymph node involvement. At the end of follow-up for this patient cohort, $20 \%$ had died from MCC. The presence of distant metastasis during the follow-up was significantly correlated to MCC specific death ( $p$-value $<0.00001)$. Immunohistochemical staining results in comparison to MCV status is presented in Table 1.

\section{ALK expression}

ALK expression was successfully analyzed in 110 tumor samples. Two of the tumors had corrupted ALK staining on the TMA slides. From the 110 tumors, 56 were strongly positive for ALK and 16 weak positive (51\% and $15 \%$ respectively). 38 (34\%) tumors were negative for ALK. (Figure 4)

We recorded clear correlation between ALK expression and MCV-positivity; From the 79 MCV-positive tumors 66 were positive for ALK (84\%) and 65\% were strong positives, while in the MCV-negative cohort only 10 of 31 tumors were ALK positive (32\%) and $16 \%$ were strong positives. This correlation ALK expression and MCV positivity was statistically significant ( $p$ 0.000006). However, ALK expression did not correlate with patient sex, tumor location or development of metastasis or MCC specific death.

This article is protected by copyright. All rights reserved. 


\section{EGFR expression}

111 tumors were analyzed for EGFR and only 7 (6.3\%) were positive for EGFR. Notably all EGFR positive tumors were MCV-negative and $22 \%$ of the $31 \mathrm{MCV}$-negative tumors expressed EGFR. ( $p 0.000011$ and 0.000077 respectively). (Figure 5) We were unable to find significant correlation with EGFR expression to sex, location, metastasis or MCC specific death. Although there was slight orientation to more severe course of disease since 3 out of 7 EGFR positive tumors developed metastasis and died from MCC. However, this observation was not statistically significant.

\section{EZH2 expression}

Our examination revealed abundant expression of EZH2 in MCC tumors. 84 (76 \%) tumors out of 111 showed strong positivity, while 18 (\%) tumors were weak positive, and only 9 (8\%) were negative. EZH2 expression was not related to tumor MCV-status or any of the clinical parameters that were analyzed.

\section{Discussion}

Herein, we studied the immunohistochemical expression of ALK, EGFR and EZH2 in 112 Merkel cell carcinoma TMA cores. We showed that ALK expression is common in MCC, although less frequent than previously reported. The intensity of ALK expression seemed to vary between the ALK positive samples. We did not establish correlation between ALK immunopositivity and clinical characteristics. However, we showed evident correlation of ALK expression and tumor MCV-positivity. The correlation was particularly apparent with 
tumors that expressed strong ALK positivity, since $91 \%$ of them were MCV-positive. It is uncharted whether ALK and MCV are interacting, but it could be that the MCV promotes ALK expression via an unknown mechanism.

We have previously studied ALK in MCC tumors and found strong expression of ALK at mRNA and protein level. ${ }^{9}$ In our previous study, we applied fluorescence in situ hybridization to seek for chromosomal translocations, but no fusions of ALK were uncovered. ${ }^{9}$ In previously conducted immunohistochemistry studies, ALK expression has been frequent in MCC, however no correlation to tumor MCV status has been reported. Filtenborg-Barnkob et al. reported ALK expression frequency as high as $93.8 \%$ on 32 MCC tumors, and our group also recorded ALK positivity in 22 of 24 MCC tumors examined $(91,7 \%)^{9,} 20$

Based on available literature, MCC tumors lack EGFR expression. ${ }^{25}$ In our previous studies we recorded under expression of EGFR at mRNA level in MCC tumors compared to normal skin ${ }^{9}$, and in keeping with other work, found no immunohistochemical EGFR positivity in any of the MCC tumors studied. Yet, we discovered EGFR mutations in $22 \%$ of 27 MCC tumors ${ }^{8}$. This encouraged us to explore the expression of EGFR in a greater tumor multitude. In this paper we demonstrated that a small number of MCC (6\%) were EGFR positive and significantly all of them were MCV-negative. We even observed possible association between EGFR positivity and metastasis development and death from MCC, but the association was not statistically significant. Since the low number of EGFR positive cases causes a challenge in analyzing correlations to clinical data, more numerous tumor cohorts are required to further assess this potential correlation. 
In contrast to our previous study where we did not find EGFR positivity in MCC tumors by IHC with the antibody clone $31 \mathrm{G7}$, which reacts with the extracellular domain of EGFR, ${ }^{8}$, we now used a different EGFR antibody, clone 5B7, which binds to the intracellular domain of EGFR and also detects truncated forms of the receptor that are constitutively active. ${ }^{26}$ The clone 5B7 has been used to detect expression of EGFR in non-small cell lung cancer TMA samples and was found to predict response to an EGFR Tyrosine Kinase inhibitor treatment. ${ }^{26,}{ }^{27}$ We therefore suggest that the small subset of MCC tumors that are EGFR positive and MCV-negative, might benefit from EGFR inhibitor treatment, and they could be identified with a relatively easy and cost effective method like IHC.

We earlier reported EZH2 expression at mRNA level in MCC tumors ${ }^{9}$. In addition, there has been activating EZH2 mutation in $1 \mathrm{MCC}$ tumor out of 15 tumors studied ${ }^{28}$, however, other mutational studies on MCC have not recorded EZH2 mutations. ${ }^{8,18,19}$ A recent study by Harms et al. suggested that EZH2 could be a prognostic factor in MCC. Higher expression of EZH2 correlated with worse 5-year MCC-specific survival. ${ }^{29}$ Correspondingly to their study, we observed frequent expression of EZH2 in MCC tumors (92\%), and the intensity of the expression varied between the tumors. Therefore, we categorized the positivity either weak or strong. However, our cohort also displayed $8 \%$ completely negative tumors. Contrary to Harms et al, we only examined primary MCC tumors, while their cohort included tumor metastases. We did not identify correlation between primary tumor EZH2 expression and development of metastasis or MCC-specific death. Therefore, usability of EZH2 as a prognostic factor is yet uncertain. However, since the majority of MCC tumors are EZH2 
positive, it could be a potential therapeutic target, and clinical trials with forthcoming EZH2 inhibitors would be recommended.

A current trend in cancer research and treatment is to go deeper into individual tumors characteristics and develop personalized treatment for cancer patients. ${ }^{30}$ Conventional drug trials conducted in large patient cohorts fail to demonstrate efficacy of treatments that only affect a certain group of patients. A particular challenge with MCC is that firstly it is rare and secondly, part of the tumors are associated with MCV and therefore embody distinct pathologic properties. Gaining knowledge of genetic and molecular alterations in MCVpositive and MCV-negative tumors poses an opportunity to determine tumors that might benefit from already existing treatments such as tyrosine kinase inhibitors, for example. Our vision is that future clinical trials for advanced MCC should take into account the individual molecular characteristics of the tumor.

We conclude that protein expression in MCV-positive and MCV-negative tumors is diverse and also differs in individual tumors. ALK expression correlates to MCV-positivity, while EZH2 expression is frequent regardless of MCV-status. A subset of MCV-negative tumors express EGFR and might respond to EGFR TKI treatment.

This article is protected by copyright. All rights reserved. 


\section{Acknowledgements}

This study received funding from Finska Läkaresällskapet, Liv och Hälsa and the Finnish cancer foundation. The funding sources were not involved in design of the study, collection, analysis and interpretation of data or writing the manuscript.

\section{References}

1. Feng $H$, Shuda $M$, Chang $Y$, Moore PS. Clonal integration of a polyomavirus in human Merkel cell carcinoma. Science 2008; 319: 1096-1100.

2. Liu W, MacDonald M, You J. Merkel cell polyomavirus infection and Merkel cell carcinoma. Curr Opin Virol 2016; 20: 20-27. DOI: S1879-6257(16)30094-3 [pii].

3. Agelli M, Clegg LX, Becker JC, Rollison DE. The etiology and epidemiology of merkel cell carcinoma. Curr Probl Cancer 2010; 34: 14-37.

4. Bhatia K, Goedert JJ, Modali R, Preiss L, Ayers LW. Merkel cell carcinoma subgroups by Merkel cell polyomavirus DNA relative abundance and oncogene expression. International Journal of Cancer 2010; 126: 2240-2246.

5. Prewett SL, Ajithkumar T. Merkel Cell Carcinoma: Current Management and Controversies. Clin Oncol (R Coll Radiol) 2015; 27: 436-444. DOI: 10.1016/j.clon.2015.04.007 [doi].

6. Moshiri AS, Doumani R, Yelistratova L, et al. Polyomavirus-Negative Merkel Cell Carcinoma: A More Aggressive Subtype Based on Analysis of 282 Cases Using Multimodal Tumor Virus Detection. J Invest Dermatol 2017; 137: 819-827. DOI: S0022-202X(16)32613-6 [pii].

7. Albores-Saavedra J, Batich K, Chable-Montero F, Sagy N, Schwartz AM, Henson DE. Merkel cell carcinoma demographics, morphology, and survival based on 3870 cases: a population based study. J Cutan Pathol 2010; 37: 20-27. DOI: 10.1111/j.1600-0560.2009.01370.x [doi].

8. Veija T, Sarhadi VK, Koljonen V, Bohling T, Knuutila S. Hotspot mutations in polyomavirus positive and negative Merkel cell carcinomas. Cancer Genet 2016; 209: 30-35. DOI: 10.1016/j.cancergen.2015.11.006 [doi].

9. Veija T, Koljonen V, Bohling T, Kero M, Knuutila S, Sarhadi VK. Aberrant expression of ALK and EZH2 in Merkel cell carcinoma. BMC Cancer 2017; 17: 236-017-3233-5. DOI:

10.1186/s12885-017-3233-5 [doi].

This article is protected by copyright. All rights reserved. 
10. Bronte G, Rizzo S, La Paglia L, et al. Driver mutations and differential sensitivity to targeted therapies: a new approach to the treatment of lung adenocarcinoma. Cancer Treat Rev 2010; 36 Suppl 3: S21-9. DOI: 10.1016/S0305-7372(10)70016-5 [doi].

11. Brandao EP, Pantarotto MG, Cruz M. A novel EGFR mutation in exon 18 with high sensitivity to EGFR TKI treatment with reduced dose. J Thorac Oncol 2012; 7: e32. DOI: 10.1097/JTO.0b013e31826d8f66 [doi].

12. Zhang $H$, Berezov $A$, Wang $Q$, et al. ErbB receptors: from oncogenes to targeted cancer therapies. J Clin Invest 2007; 117: 2051-2058. DOI: 10.1172/JCl32278 [doi].

13. Oda K, Matsuoka Y, Funahashi A, Kitano H. A comprehensive pathway map of epidermal growth factor receptor signaling. Molecular Systems Biology 2005; 1: 2005.0010-2005.0010. DOI: $10.1038 / \mathrm{msb} 4100014$.

14. Liu J, Jin $\mathrm{H}$, Tian $\mathrm{H}$, et al. Anaplastic lymphoma kinase protein expression predicts micrometastases and prognosis for patients with hepatocellular carcinoma. Oncol Lett 2016; 11: 213-223. DOI: 10.3892/ol.2015.3859 [doi].

15. Murugan AK, Xing M. Anaplastic thyroid cancers harbor novel oncogenic mutations of the ALK gene. Cancer Res 2011; 71: 4403-4411. DOI: 10.1158/0008-5472.CAN-10-4041 [doi].

16. Tuononen K, Sarhadi VK, Wirtanen A, et al. Targeted resequencing reveals ALK fusions in non-small cell lung carcinomas detected by FISH, immunohistochemistry, and real-time RTPCR: a comparison of four methods. Biomed Res Int 2013; 2013: 757490. DOI: $10.1155 / 2013 / 757490$ [doi].

17. Morris SW, Kirstein MN, Valentine MB, et al. Fusion of a kinase gene, ALK, to a nucleolar protein gene, NPM, in non-Hodgkin's lymphoma. Science 1994; 263: 1281-1284.

18. Goh G, Walradt T, Markarov V, et al. Mutational landscape of MCPyV-positive and MCPyV-negative Merkel cell carcinomas with implications for immunotherapy. Oncotarget 2016; 7: 3403-3415. DOI: 10.18632/oncotarget.6494 [doi].

19. Harms PW, Vats $P$, Verhaegen ME, et al. The Distinctive Mutational Spectra of Polyomavirus-Negative Merkel Cell Carcinoma. Cancer Res 2015; 75: 3720-3727. DOI: 10.1158/0008-5472.CAN-15-0702 [doi].

20. Filtenborg-Barnkob BE, Bzorek M. Expression of anaplastic lymphoma kinase in Merkel cell carcinomas. Hum Pathol 2013; 44: 1656-1664. DOI: 10.1016/j.humpath.2012.11.021 [doi].

21. Kim KH, Roberts CW. Targeting EZH2 in cancer. Nat Med 2016; 22: 128-134. DOI: 10.1038/nm.4036 [doi].

22. Italiano A, Soria JC, Toulmonde M, et al. Tazemetostat, an EZH2 inhibitor, in relapsed or refractory B-cell non-Hodgkin lymphoma and advanced solid tumours: a first-in-human, open-label, phase 1 study. Lancet Oncol 2018; 19: 649-659. DOI: S1470-2045(18)30145-1 [pii].

23. Wong SQ, Waldeck K, Vergara IA, et al. UV-Associated Mutations Underlie the Etiology of MCV-Negative Merkel Cell Carcinomas. Cancer Res 2015; 75: 5228-5234. DOI:

10.1158/0008-5472.CAN-15-1877 [doi].

This article is protected by copyright. All rights reserved. 
24. Sihto H, Kukko H, Koljonen V, Sankila R, Bohling T, Joensuu H. Clinical factors associated with Merkel cell polyomavirus infection in Merkel cell carcinoma. J Natl Cancer Inst 2009; 101: 938-945.

25. Brunner $M$, Thurnher D, Pammer J, et al. Expression of VEGF-A/C, VEGF-R2, PDGFalpha/beta, c-kit, EGFR, Her-2/Neu, Mcl-1 and Bmi-1 in Merkel cell carcinoma. Mod Pathol 2008; 21: 876-884. DOI: 10.1038/modpathol.2008.63 [doi].

26. Mascaux C, Wynes MW, Kato Y, et al. EGFR Protein Expression in Non-Small Cell Lung Cancer Predicts Response to an EGFR Tyrosine Kinase Inhibitor â $€$ " A Novel Antibody for Immunohistochemistry or AQUA Technology. Clin Cancer Res 2011; 17: 7796-7807. DOI: 10.1158/1078-0432.CCR-11-0209 [doi].

27. Chang $\mathrm{H}$, Oh J, Zhang $\mathrm{X}$, et al. EGFR protein expression using a specific intracellular domain antibody and PTEN and clinical outcomes in squamous cell lung cancer patients with EGFR-tyrosine kinase inhibitor therapy. Onco Targets Ther 2016; 9: 5153-5162. DOI: 10.2147/OTT.S107291 [doi].

28. Harms PW, Collie AM, Hovelson DH, et al. Next generation sequencing of Cytokeratin 20negative Merkel cell carcinoma reveals ultraviolet-signature mutations and recurrent TP53 and RB1 inactivation. Mod Pathol 2016; 29: 240-248. DOI: 10.1038/modpathol.2015.154 [doi].

29. Harms KL, Chubb H, Zhao L, et al. Increased expression of EZH2 in Merkel cell carcinoma is associated with disease progression and poorer prognosis. Hum Pathol 2017; 67: 78-84. DOI: S0046-8177(17)30259-9 [pii].

30. Jackson SE, Chester JD. Personalised cancer medicine. Int J Cancer 2015; 137: 262-266.

DOI: 10.1002/ijc.28940 [doi].

This article is protected by copyright. All rights reserved. 
Table 1. Number and percentages of MCV-negative and MCV-positive tumors with certain staining result.

\section{MCV}

\begin{tabular}{|l|lll}
\hline ALK & Negative & Positive & Total \\
- STRONG & $5(9 \%)$ & $51(91 \%)$ & 56 \\
- WEAK & $5(31 \%)$ & $11(69 \%)$ & 16 \\
$\begin{array}{l}\text { - NEGATIVE } \\
\text { TOTAL }\end{array}$ & $21(55 \%)$ & $17(45 \%)$ & 38 \\
EGFR & $31(28 \%)$ & $79(72 \%)$ & 110 \\
- POSITIVE & $7(100 \%)$ & $0(0 \%)$ & 7 \\
- NEGATIVE & $24(23 \%)$ & $80(77 \%)$ & 104 \\
TOTAL & $31(28 \%)$ & $80(72 \%)$ & 111 \\
EZH2 & & & \\
- STRONG & $22(26 \%)$ & $62(74 \%)$ & 84 \\
- WEAK & $6(33 \%)$ & $12(67 \%)$ & 18 \\
- NEGATIVE & $3(33 \%)$ & $6(67 \%)$ & 9 \\
TOTAL & $31(28 \%)$ & $80(72 \%)$ & 111 \\
\hline
\end{tabular}

\section{Figure legends}

Figure 1: ALK expression by immunohistochemistry. Upper panel shows strong positive staining while lower panel represents weak positive staining. 200x magnification.

Figure 2: EGFR expression by immunohistochemistry. Upper panel shows positive staining while lower represents apparent negative staining. 200x magnification.

Figure 3: EZH2 expression by immunohistochemistry. Upper panel shows strong positive staining while lower panel represents weak positive staining. 200x magnification.

Figure 4: Frequency of ALK positive and negative MCC samples in comparison to MCV status. Strong positives are marked "+" and weak negatives " $(+)$ ".

Figure 5: Frequency of EGFR positive and negative MCC samples in comparison to MCV status. 



This article is protected by copyright. All rights reserved. 

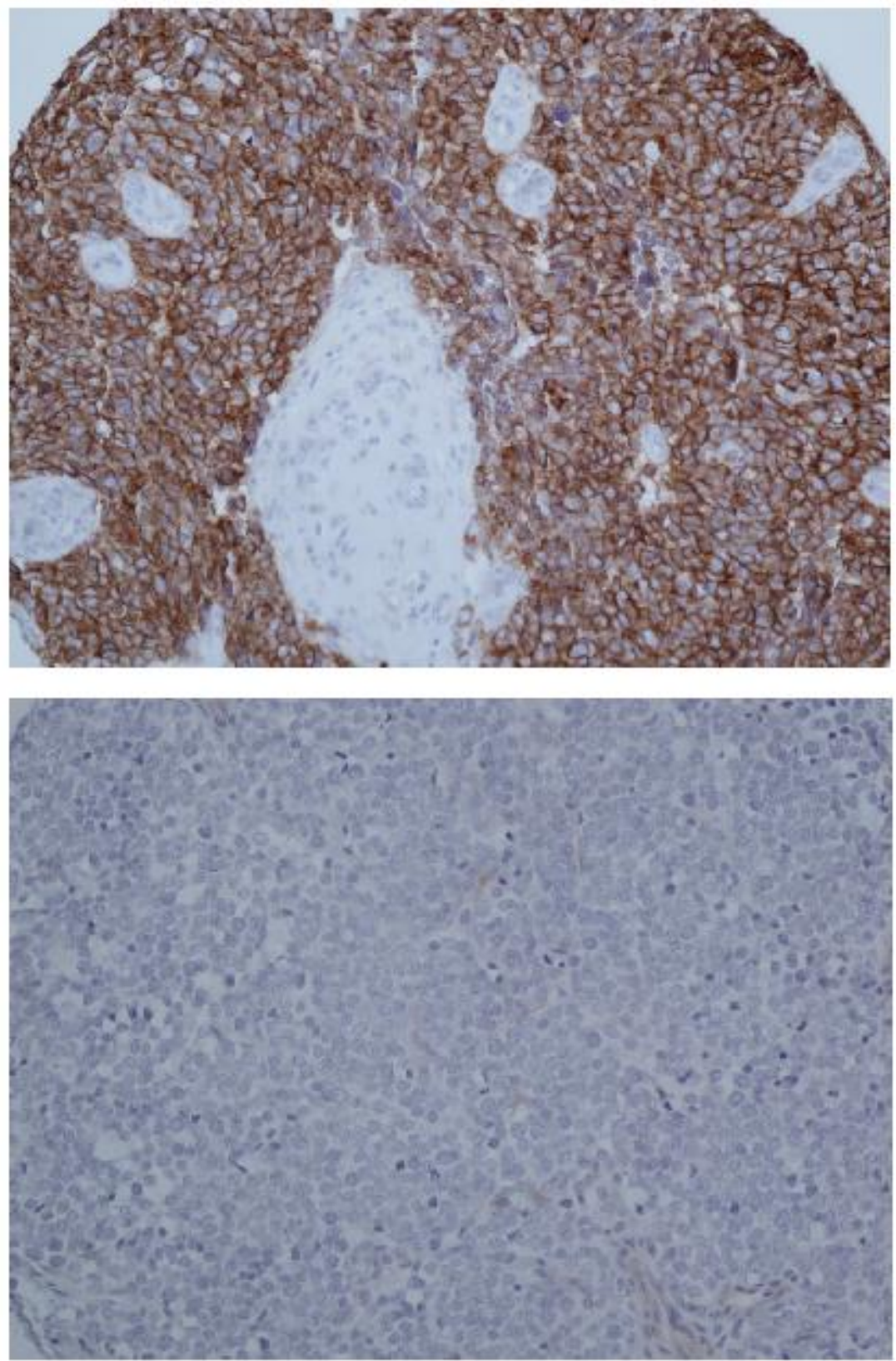

This article is protected by copyright. All rights reserved. 



This article is protected by copyright. All rights reserved. 




This article is protected by copyright. All rights reserved. 


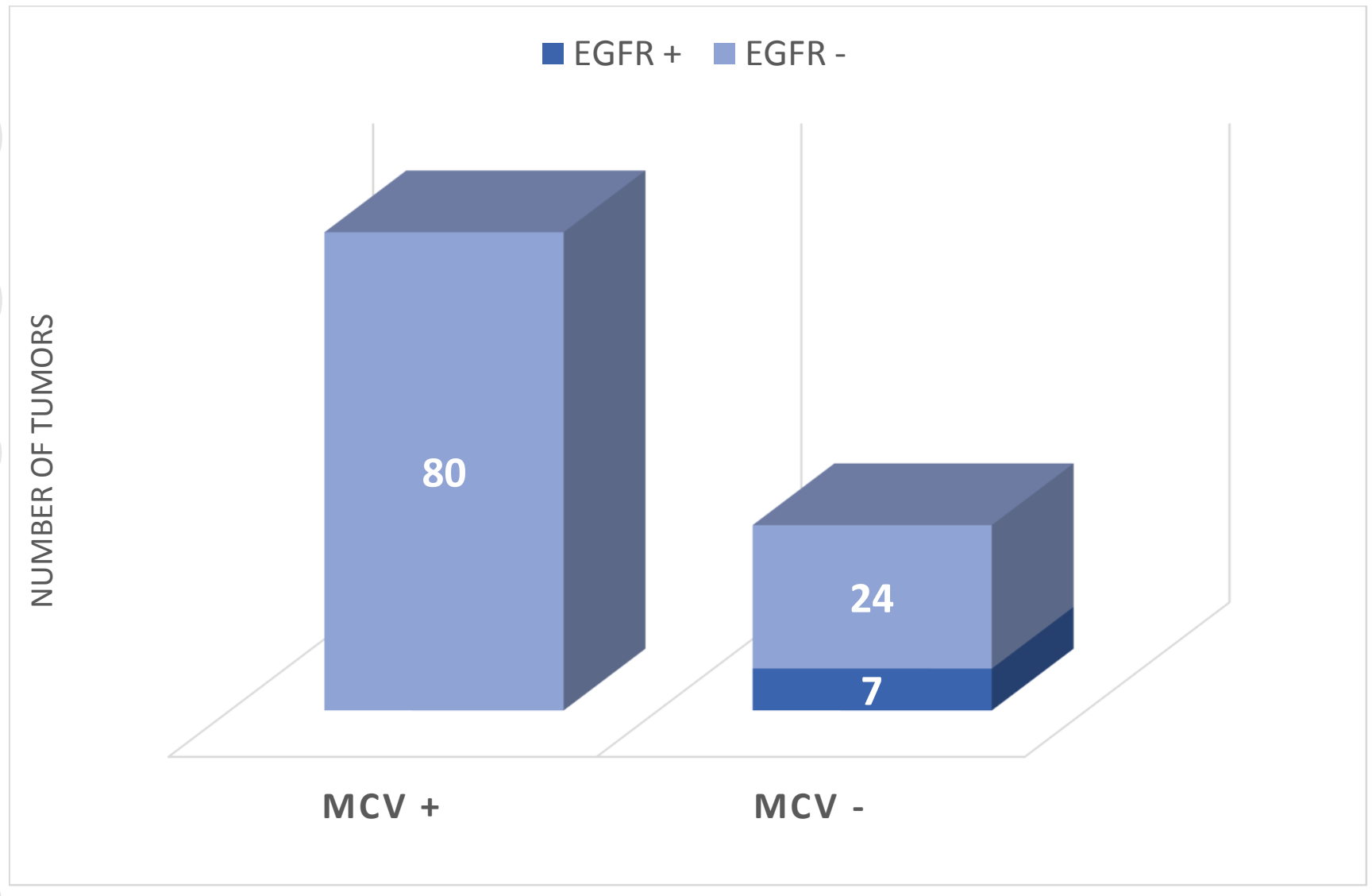

This article is protected by copyright. All rights reserved. 